\title{
VEMUS: An Integrated Platform to Support Music Tuition Tasks
}

\author{
George Tambouratzis, Kostas Perifanos, Iro Voulgari \\ Institute for Language and Speech Processing, Artemidos 6, 15125 Athens, Greece \\ giorg_t@ilsp.gr, kperi@ilsp.gr, voulgari@di.uoa.gr \\ Anders Askenfelt, Svante Granqvist, Kjetil Falkenberg Hansen \\ KTH - Royal Institute of Technology, Dept. of Speech, Music and Hearing, \\ Stockholm 10044, Sweden \\ andersa@speech.kth.se, svante@speech.kth.se, hansen@speech.kth.se \\ Yann Orlarey, Dominique Fober, Stephane Letz \\ GRAME - Centre Nationale de Creation Musicale, 9 Rue de Garet, 69202 Lyon, France \\ orlarey@grame.fr, fober@grame.fr, letz@grame.fr
}

\begin{abstract}
In this paper, the VEMUS platform is presented, as a novel approach for music tuition that focuses on beginner and intermediate students, typically aged from 9 to 15 years. This platform is characterized by an open, highly interactive and networked multilingual music tuition framework that covers a selection of popular wind instruments. The VEMUS environment integrates innovative, pedagogically-motivated $e$ learning components to augment traditional music teaching in three distinct learning settings, namely self-practicing, classroom and distance learning. In the present article, the current stage of development of VEMUS is presented, and the areas where it might be of most use towards supporting the educational activities associated with music tuition are identified.
\end{abstract}

\section{Introduction}

Music education through the use of ICT seems to be a demanding and multifaceted process involving both the instructional methodology and approach of the teacher as well as the involvement, participation and learning from the part of the students [1], [2], [3], [4]. Extensive research has been carried out on the impact of ICT in music education as well as commercial development of software for music composition targeted at a range of users from novice students to professional musicians. However, research on music education support tools integrating specific educational methodologies has been limited. The VEMUS Music
School, developed within the framework of the ongoing VEMUS (Virtual European Music School) EU project addresses the issue with the development of a holistic environment for the support of teachers and students. Based on the findings and platform developed during the IMUTUS project [5], [6], an interactive learning platform was developed. This platform integrates tools that augment teaching practices for selected wind instruments, providing a much more accurate evaluation of the student performance and covering a larger number of environments, while providing feedback via different yet complementary avenues.

Here, the VEMUS platform is described in detail, focusing on the manner in which it assists the learning process. In Section 2, the principles of the platform and the learning environments it covers are presented. Details regarding the VEMUS implementation are presented in Section 3. The content aspect of VEMUS is summarized in Section 4. In Section 5, details on using VEMUS are described, while issues related to the platform evaluation are treated in Section 6.

\section{VEMUS Principles}

VEMUS aims at developing an open, highly interactive and networked multilingual music tuition framework covering a number of popular wind instruments. Though VEMUS builds on core technological tools developed in IMUTUS, it extends the pedagogical and technological basis of IMUTUS by covering additional instruments, integrating a wider 
range of functionalities and supporting enhanced pedagogical scenarios.

The VEMUS platform integrates innovative elearning components addressing three learning settings: (i) a self-practicing environment that focuses on automatic performance evaluation and feedback generation to maximise the practicing effectiveness, (ii) innovative tools for teaching music in groups of students by supporting collaborative activities, and (iii) a distance learning platform that couples a content repository with communication and progressmonitoring tools, for remotely coaching students.

The platform is aimed at novice students aged 9-15 and adopts a holistic approach for the support of the instructional and learning processes. By exploiting the potential of ICT it aims at triggering the motivation of the students to spend more time with the educational material, supporting both independent and collaborative practice. More specifically, VEMUS provides the possibility to practice on specific musical pieces and hear reference recordings, to record student performances, and submit these for evaluation and to provide customized assessment/feedback to the students [7]. These functionalities are integrated in a consistent framework that does not interfere with the musical experience of either the teachers or students.

A survey associated to this project has indicated that there exists a need for such a functionality. $88 \%$ of the responding students have access to a computer at home, while $69 \%$ of them believe that the computer could assist them in studying music and are willing to use a platform such as VEMUS. VEMUS adopts an international dimension via the participation of 8 European partners (from Greece, France, Sweden, Lithuania, Estonia and Romania) and integrates elearning activities in various contexts within a unified platform.

\section{Platform Description}

\subsection{Self-practicing environment}

The VEMUS self-practicing environment implements a virtual 'assistant teacher' concept for guidance when the student practices at home, giving additional feedback between sessions with the actual teacher [8]. Automatic performance evaluation and structured feedback are provided, tailored to each student in order to maximise the effectiveness of selfpracticing, by giving both general and detailed comments on the student performance.

The VEMUS feedback after each performance is restricted to a selection of pedagogically prioritized errors/mistakes. These are coupled to the VEMUS
Music Schoolhouse concept, which allows the teacher to adapt VEMUS to the specific student's level and character. More specifically, the Music Schoolhouse concept involves a number of floors as well as virtual teachers of different strictness levels, to provide an adaptable system feedback strategy.

In addition, the pedagogic approach developed by VEMUS is based on an extended music score, intended to facilitate the learning process by means of new types of feedback. This approach could be summarized with a simple metaphor: the music score behaves like a visual and aural mirror, helping the student in getting a better consciousness of his instrumental performance.

\subsection{Music classroom environment}

VEMUS explores innovative tools to support music teaching in group settings, these ranging from aids that assist the teacher in preparing a classroom assignment to tools that support collaborative learning and group activities. These allow the introduction of VEMUS to the classroom, as shown in Figure 1, to enhance the music tuition processes.

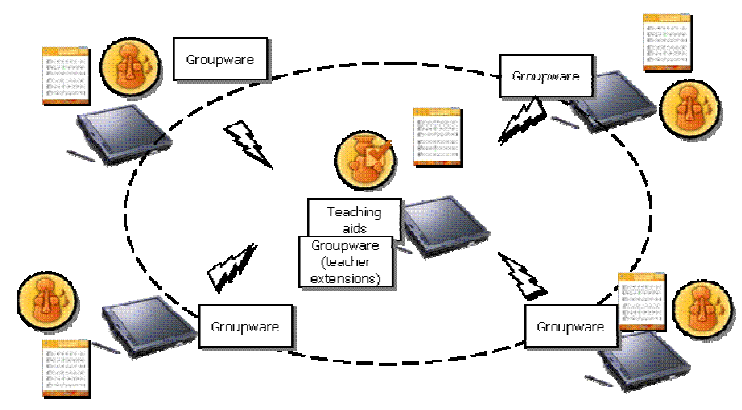

Figure 1: The VEMUS classroom environment interconnecting students and their teacher.

A main aspect is the ability of the user to communicate directly with other VEMUS users, both teachers and students, via his/her computer. This is achieved via a custom-built VEMUS networking and configuration manager. This manager uses the wireless capabilities of modern laptops and tablet PCs and a peer-to-peer solution to create a network connecting the users' computers, without requiring a custom configuration. Using this facility, students within a classroom, coordinated by their teacher, may actively collaborate and interact, exchanging music scores, performances, comments and annotations. This engagement into collaborative activities leads to the enhancement of the students' learning experience. It provides teachers with new capabilities for organising 
the learning material within the classroom and forms a major research point of the VEMUS evaluation.

\subsection{Distance learning environment}

Although the effects of distance learning in education have been studied, the topic of distance learning in the field of music education has not yet been fully investigated. Still, an increased number of complete courses and entire degree programs in music education are now becoming commonplace [9].

The VEMUS platform incorporates a distance learning environment (DLE) to maintain and manage an open content repository. This repository stores a collection of music exercises and assignments, intended to enable the improvement of the student skills. At the same time, authoring and communication tools are provided that allow the introduction of new music material by the teacher and the remote coaching of students, while supporting the monitoring of their progress through time. The DLE enables the participation to music tuition activities of students that might otherwise be prohibited due to geographic or other constraints. Furthermore, the DLE activation extends the relation of teachers and learners beyond the physical limits and time schedules of the classroom.

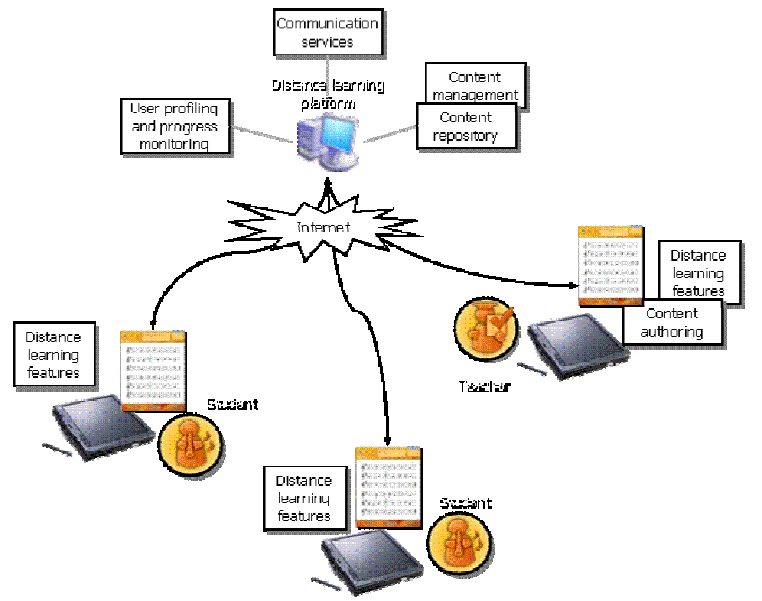

Figure 2: Diagram of the connection between teacher and students via the VEMUS DLE.

In contrast to the proprietary IMUTUS distancelearning platform, VEMUS focuses on a clean-sheetof-paper approach with substantially enhanced functionality. Following a review of the available technology, it was decided to use the MOODLE opensource platform [10]. The main features of MOODLE support a large subset of the VEMUS DLE specifications, as defined by the user requirements. New courses and tools have been developed and the DLE platform is being adapted to the project objectives, while the interface and layout are designed to be consistent with the complementary VEMUS modules.

\subsection{Selecting the VEMUS music instruments}

As concerns the instruments to be covered by VEMUS, a survey was performed regarding music instrument popularity throughout the countries of the VEMUS consortium. The survey purpose was to identify the most widely taught wind instruments at a European level in order to maximise the potential audience of VEMUS. The most popular wind instruments have been found to be, in decreasing order of popularity, (i) the flute, (ii) the saxophone, (iii) the clarinet and (iv) the trumpet. As the recorder is quite popular, it has also been chosen for VEMUS.

\section{Using the VEMUS Platform}

VEMUS relies on the active participation of music teachers. Regular face-to-face lessons with a teacher on a weekly basis provide the foundation of the concept, and teachers' experience and expertise are embedded in the different VEMUS modules.

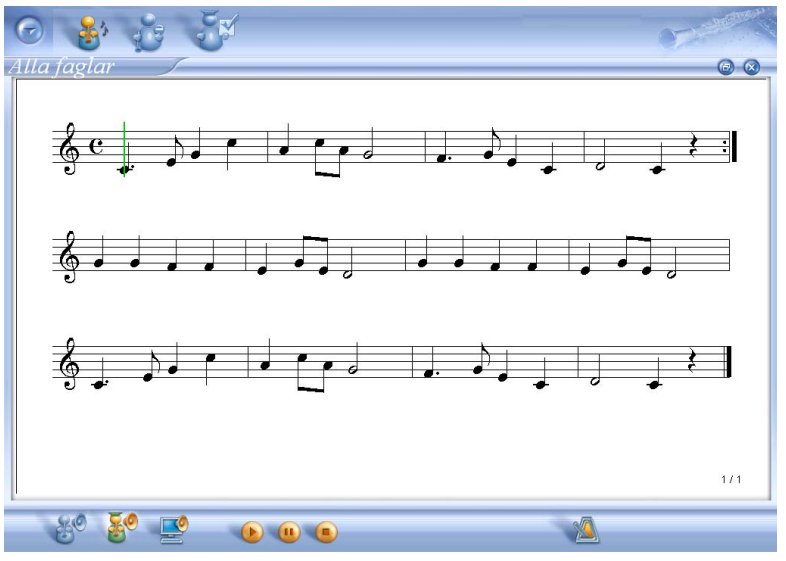

Figure 3: The main interface of the VEMUS platform for practicing a music piece.

VEMUS is intended to provide coaching and support when the actual teacher is not present and generate the necessary feedback [8] regarding the student performance. As such, VEMUS allows a student to load a music score from the available repertory, as shown in Figure 3. Then, the student performs the score and the system automatically generates customized and focused feedback in a number of graphical and visual ways. The first method is to evaluate the performance via the Performance 
Evaluation Module (PEM) [6], providing feedback in the form of short comments as indicated in Figure 4. In comparison to IMUTUS, the VEMUS PEM comprises new music note recognition engines that provide a substantially improved accuracy. The evaluation of a given performance is based on the PEM searching for mistakes using error categories proposed by music teachers. The feedback messages in VEMUS are student-centred, textual, graphical and visual and have been compiled and formulated in cooperation with specialized teachers.

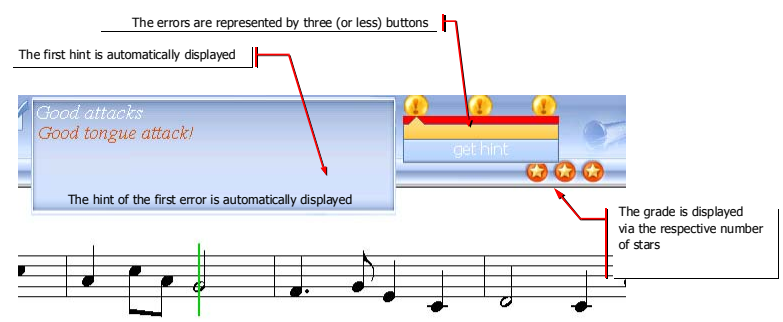

Figure 4: Sample VEMUS PEM feedback to the student following a music performance.

VEMUS also offers new pedagogical tools for the teacher based on augmenting the digitally stored scores via teacher annotations, both graphical and audio. The teacher is able to customise the homework for each student by adding appropriate annotations in the score. Via control annotations (that are invisible to the student) the teacher can select difficult score passages, on which VEMUS performance evaluation is to focus.

A student performance is recorded and associated automatically to the corresponding notes of the music score. The music score in VEMUS has been extended to play the role of user interface for accessing this recording: using the score, the student can listen to his performance, or access any location of the recording, listen to a single note or to a part of the score. Hence, it may be considered that the music score is extended to become a "sound mirror".

The VEMUS pedagogic approach is based on the extended music score concept that provides novel means to convey feedback and thus assist the learning process. Feedback is allowed on the music score by supporting the addition of graphic shapes, text, emoticons and hand-written as well as sound annotations [5]. This approach includes various graphic representations of the music performance, displayed as feedback curves in parallel to the score. These visualize the students' performances and explain hardto-teach concepts such as attacks and articulation by comparison to a reference performance.

To that end, a number of parametric feedback curves for visualising particulars of a student's performance have been defined [11]. An indicative feedback curve is shown in Figure 5. A web survey has been carried out to define which curves are the most descriptive ones, taking into account the views of both teachers and students. The most effective curves have been included in VEMUS for further investigation via field tests, to establish the pedagogic merits of each one of them.

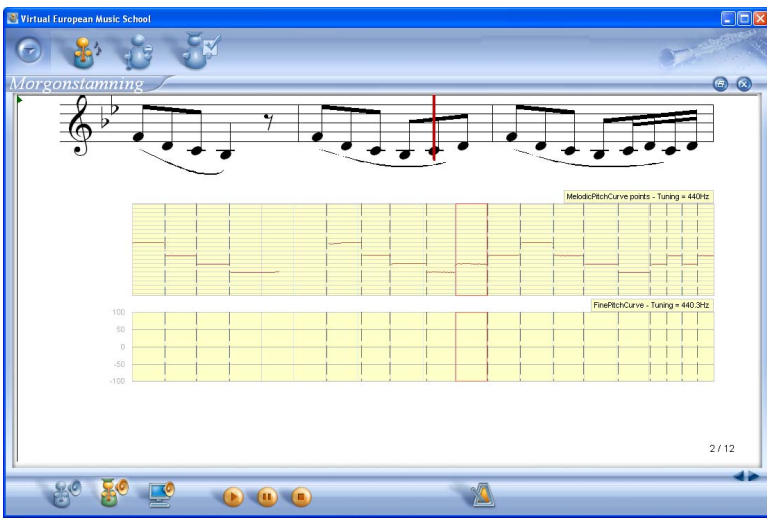

Figure 5: Advanced feedback curve example.

Finally, the VEMUS environment provides a dedicated tool that displays on the tablet/laptop screen the 3D-fingering sequence required to perform the given musical score (or a portion of the score). This assists the student in learning the needed fingerings for the given combination of instrument and music piece. The user is able to customise the view point and zoom of the instrument, by using the tablet/laptop mouse.

\section{VEMUS Content}

Within the VEMUS platform, a content collection is integrated. This comprises repertory pieces and exercises, as well as non-interactive exercises focusing on sound production and warm-up, defined by expert professors, for each one of the main instruments. Based on their teaching and pedagogical experience, these teachers have collected material reflecting both international and domestic music literature. In accordance to the VEMUS Music Schoolhouse concept, the pieces/exercises are divided in 5 main levels that correspond to Schoolhouse levels. In each level, the repertory pieces and exercises are organized into lesson groups for an even more accurate characterization of the required skills for each item.

These collected scores have been digitized and stored in a MusicXML format. Furthermore, for each music score, a dedicated structure is defined in VEMUS to store both metadata (regarding the score characteristics) as well as the actual student 
performances. Custom-built tools enable the teacher to introduce and appropriately annotate new content.

\section{Evaluation of the VEMUS platform}

The evaluation procedure for VEMUS comprises field studies involving music schools. Evaluation activities are taking place in schools in Sweden, Greece, Romania and Lithuania, complemented by validation activities in Estonia. Paired groups of students are used to determine the effect of using VEMUS during tuition compared to control students.

To evaluate the entire range of novel characteristics, a layered approach has been decided upon, with different aspects of the system being investigated in distinct stages of the evaluation process. Evaluation has focused on the quality of the student performances as well as the student's motivation in devoting more time to practicing. The VEMUS evaluation activities are currently ongoing. Initial results have shown a substantially increased motivation of VEMUS students to practice, in comparison to control students.

\section{Conclusions}

Within the framework of the VEMUS project, a holistic music instruction and learning environment integrating a number of innovative technologies has been presented. This focuses on supporting collaborative technologies that allow students in a classroom or an off-site setting to interact in a variety of ways, to the benefit of the learning process. Additionally, new graphic-based representations of the music quality are investigated, to determine those that convey the most information to the student. The student audience addressed by VEMUS is widened via the provision of a comprehensive distance learning environment that incorporates a content repository and the coverage of a multitude of popular music instruments.

It is expected that the proposed approach will effectively support teachers and students throughout the music instruction process and provide a context of interaction with peers as well as with the material, seamlessly and without interfering with the musical experience. The design and educational approach integrated in the VEMUS platform are relevant to the actual user needs and requirements, while the flexibility of the platform is expected to facilitate its effective integration into different educational settings.

\section{Acknowledgements}

This work is partially supported by European Community under the Information Society Technology (IST) RTD programme (FP6-IST-27952). The authors are solely responsible for the content of this communication. It does not represent the opinion of the European Community, and the European Community is not responsible for any use that might be made of data appearing therein.

\section{References}

[1] C. Byrne, and R.A.R. MacDonald, "The Use of Information and Communication Technology (I\&CT) in the Scottish Music Curriculum: a Focus Group Investigation of Themes and Issues", Music Education Research, Vol. 4, No. 2, pp. 263-273, 2002. ISSN 1461-3808

[2] J. Mills, and A. Murray, "Music Technology Inspected: Good Teaching in Key Stage 3", British Journal of Music Education, Vol. 17, pp. 129-156, 2000.

[3] B. Crow, "Music-related ICT in education", in C. Philpott (Ed.), Learning to Teach Music in the Secondary School, pp. 135-162, 2001. London: Routledge/Falmer.

[4] J.R. Austin, and M. Haefner Berg, "Exploring Music Practice among Sixth-Grade Band and Orchestra Students", Psychology of Music, Vol. 34, No. 4, pp. 535-558, 2006.

[5] D. Fober, S. Letz, Y. Orlarey, A. Askenfelt, K. Falkenberg Hansen, and E. Schoonderwaldt, "IMUTUS - an Interactive Music Tuition System", Proceedings of the SMC'04 Conference, pp. 97-103, 2004. IRCAM.

[6] S. Raptis, A. Chalamandaris, A. Baxevanis, A. Askenfelt, E. Schoonderwaldt, K. Falkenberg Hansen, D. Fober, S. Letz, and Y. Orlarey, "IMUTUS - an Effective Practicing Environment for Music Tuition", ICMC-2005 Conference Proceedings, pp. 383-386, 2005.

[7] A. Johnston, S. Amitani, and E. Edmonds, "Amplifying Reflective Thinking in Musical Performance", Proceedings of the $5^{\text {th }}$ Conference on Creativity \& Cognition, pp. 166175, April 2005.

[8] S. Pitts, J. Davidson, and G. McPherson, "Developing Effective Practise Strategies: Case Studies of Three Young Instrumentalists", Music Education Research, Vol. 2, No. 1, pp. 45-56, 2000.

[9] P.R. Webster, "Computer-based Technology and Music Teaching and Learning: 2000-2005", International Handbook of Research in Arts Education, pp. 1311-1328, L. Bresler (Ed.), Springer, 2007.

[10] http:// moodle.com

[11] D. Fober, S. Letz, and Y. Orlarey, "VEMUS - Feedback and Groupware Technologies for Music Instrument Learning", Proceedings of the $4^{\text {th }}$ International Music Conference, Lefkada, Greece, July 2007. 